\title{
Throughput Enhancement in the Design of Adaptive Receiver Transmission Protocol in Wireless Adhoc Network
}

\author{
Anu Priya C V ${ }^{1}$, Khaleelur Rahiman $\mathrm{P} \mathrm{F}^{2}$ \\ ${ }^{\text {" Hindusthan college of engineering and technology Coimbatore) }}$ \\ ${ }^{2(A s s i s t a n t ~ P r o f e s s o r ~ H i n d u s t h a n ~ c o l l e g e ~ o f ~ e n g i n e e r i n g ~ a n d ~ t e c h n o l o g y ~ C o i m b a t o r e) ~}$
}

\begin{abstract}
In case of decentralized coordinator the design of medium access control (MAC) protocols is considered crucial for throughput enhancement in the wireless ad hoc networks. Ad hoc network means it does not have a pre-existing infrastructure. The receiver blocking problem, which has not been studied in most of the MAC protocol design, can lead to severe degradation on the throughput performance. In this paper, the multiple receiver transmission (MRT) and the fast NAV truncation (FNT) mechanisms are proposed to alleviate the receiver blocking problem without the adoption of additional control channels. The adaptive receiver transmission(ART) scheme is proposed to further enhance the throughput performance with dynamic adjustment of the selected receivers. Analytical model is also derived to validate the effectiveness of the proposed ART protocol. Simulations are performed to evaluate and compare the proposed three protocols with existing MAC schemes can be observed that the proposed ART protocol outperforms the other Schemes by both alleviating the receiver blocking problem and enhancing the throughput performance for the wireless multihop ad hoc networks.
\end{abstract}

\section{Wireless Ad Hoc Network}

\section{Introduction}

A wireless multihop network (WMN) adopts wireless communication technologies to Maintain Connectivity and exchange messages between decentralized nodes in the multihop Manners. This type of wireless networks is capable to perform self-creating, administering, and organizing the network connectivity. With the decentralized characteristics of the WMNs, feasible design of medium access control (MAC) protocol is considered important for performance enhancement. However, the connectivity between the network nodes is in general not guaranteed in the WMN, Which incurs notorious exposed node and hidden node problems. Some early attempts for resolving these problems suggested the usage of request-to-send (RTS) and clear-to send (CTS) mechanisms, which were later adopted by the 802.11 MAC protocol suite can be employed in the WMNs since it has been specified to support Decentralized operations called the ad hoc mode.

\section{MAC Protocol}

In the seven-layer OSI model of computer networking, media access control (MAC) data communication protocol is a sub layer of the data link layer, which itself is layer 2. The MAC sub layer provides addressing and channel access control mechanisms that make it possible for several terminals or network nodes to communicate within a multiple access network that incorporates a shared medium, e.g. Ethernet. The hardware that implements the MAC is referred to as a medium access controller. The MAC sub layer acts as an interface between the logical link control (LLC) sub layer and the network's physical layer. The MAC layer emulates a full-duplex logical communication channel in a multi-point network. This channel may provide uncast, multicast or broadcast communication service

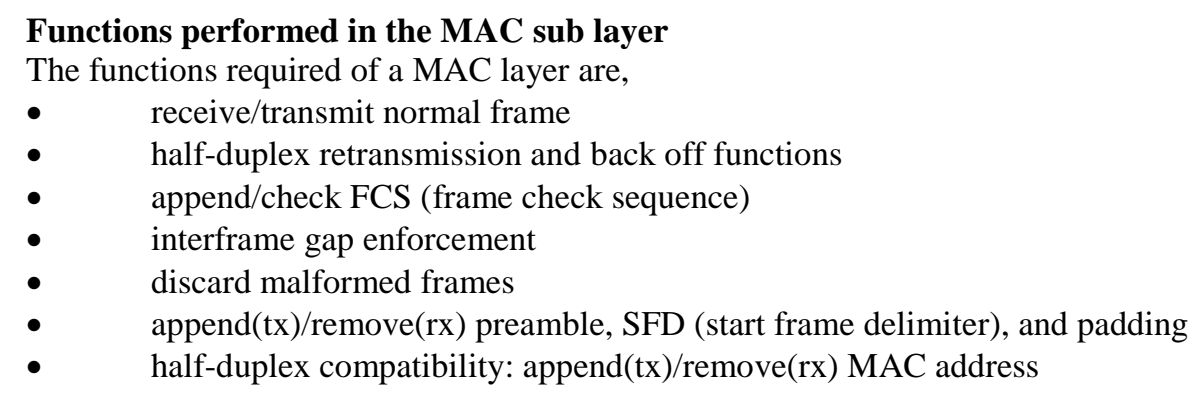




\section{Implementation Phase \\ Existing Scheme}

In this project, the multiple receiver transmission (MRT) and the fast NAV truncation (FNT) mechanisms are proposed to cope with the receiver blocking problem without adopting either additional control channels or transceivers. The MRT approach is proposed to provide additional opportunities for the transmission to multiple receivers; while the FNT scheme reduces the duration of the network allocation vector (NAV) to provide channel accessing opportunities for the other nodes in the network. Note that the MRT scheme is designed with the scenario that a node has data ready for multiple one-hop neighboring receivers, which is consider common and feasible especially for the sensor networks. Each sensor can monitor the environment and exchange its message with its neighbor nodes. However, both the MRT and FNT schemes may suffer performance degradation under specific network scenarios. For the MRT approach, a large amount of CTS packets from those multiple receivers are required to provide extra opportunities for data transmission. Considering more than one receivers replying the CTS packets, the data transmission delay of these selected receivers will be increased because each receiver has to wait for the data packets that are not destined to itself until the end of entire transmission . Moreover, the FNT mechanism does not provide additional transmission opportunity for multiple receivers, which leads to limited performance improvement. Therefore, considering the drawbacks from the MRT and FNT schemes, an adaptive receiver transmission (ART) protocol is proposed to further enhance the network efficiency and channel utilization. The analytical model for throughput performance of the proposed ART protocol will be derived and further validated with simulations. The performance evaluation of the proposed schemes will be performed and compared with the conventional IEEE 802.11a DCF protocol and other existing schemes via simulations. It will be shown that the receiver blocking problem can be effectively alleviated with the adoption of proposed MRT, FNT, and ART schemes. The network throughput can consequently be enhanced.

\section{Network Model And Blocking Problem}

Considering a set of nodes $\mathrm{N}=\{\mathrm{Ni} /$ for all $\mathrm{i}\}$ within a two dimensional euclidean plane, the locations of the set $\mathrm{N}$ are represented by the set $\mathrm{P}=\{\mathrm{PNi} / \mathrm{PNi}=(\mathrm{xNi}, \mathrm{yNi}), \forall \mathrm{i}\}$.It is assumed that all the nodes are homogeneous and equipped with omnidirectional antennas under a single channel. The set of close disks defining the transmission ranges of $\mathrm{Ni}$ in $\mathrm{N}$ is denoted as The receiver blocking problem associated with the receiver blocking group are defined as follows:

Definition 1 (Receiver blocking group) :- Given the set $\mathrm{S} \subseteq \mathrm{N}$, which includes all the transmitters and receivers, the receiver blocking group is defined as, $\mathrm{BS}=\mathrm{UNi} \in \mathrm{sTNi}$, because all the nodes in $\mathrm{BS}$ are blocked either by the carrier sensing mechanisms or due to the on-going packet transmission.

Problem 1 (Receiver blocking problem):- Let BS be the receiver blocking group within the network. The receiver blocking problem occurs while a node $\mathrm{Ni} E(\mathrm{~N}-\mathrm{BS})$ intends to communicate with a node $\mathrm{Nj} \varepsilon \mathrm{BS}$. Due to the blocking nature of $\mathrm{Nj}$, a large amount of useless connection request packets will be issued by $\mathrm{Ni}$, which leads to the degradation of network throughput.

Fig. 3.1 illustrates the schematic diagram for the receiver blocking problem with the network topology and the corresponding timing diagram.

As shown in Fig.1a, it is considered that $\mathrm{N} 1$ and $\mathrm{N} 2$ constitute the on-going transmission pair as identified by the solid arrow, i.e., $\mathrm{S}=(\mathrm{N} 1, \mathrm{~N} 2)$. The receiver blocking problem happens if NA $\varepsilon(\mathrm{N}-\mathrm{BS})$ intends to initiate a communication link with $\mathrm{N} 3 \varepsilon$ BS, i.e., denoted by the dashed arrow. Based on Definition 1, the receiver blocking group is obtained as $\mathrm{BS}=(\mathrm{N} 1 \ldots \mathrm{N} 9)$, which lies within the light gray region as in Fig.3.1a.

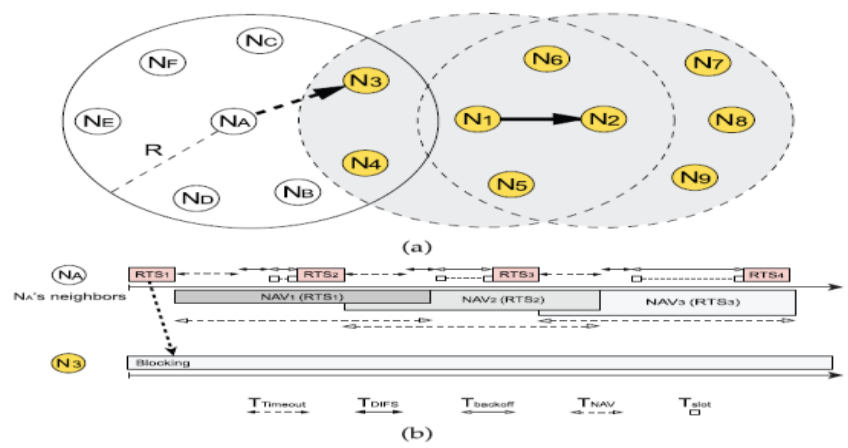

Fig 1 Schematic diagram for the receiver blocking problem 
Note that the receiver blocking problem will not occur if both nodes that intend to communicate are located in BS. Referring to Fig. 3.1b, NA will attempt to communicate with N3 by transmitting the RTS Packet (i.e.,RTS1) after the successful channel contention. Based on the broadcast nature, NB and NC will also receive the RTS1 packet and consequently set up their corresponding NAV timers to refrain from accessing the channel, i.e.,TNAV = TCTS + TData + TACK + 3TSIFS + 3Tprop. It is noted that the subscript in each timing parameter is utilized to denote its corresponding meaning, i.e., TCTS, TData, TACK, TSIFS, and Tprop indicate the time durations for the CTS packet, data packet, ACK packet, the short interframe space, and the propagation delay, respectively. Moreover, Tslot and TDIFS in Fig. $1 \mathrm{~b}$ represent the slot time of conventional IEEE 802.11 standard and the time duration for the DCF interframe space, respectively; while the parameter Tbackoff indicates the time interval for the current back off window of a node.

However, N3 will not respond to the RTS1 packet with a corresponding CTS packet due to the PCS/VCS mechanisms. After a time-out Ttimeout =TCTS + TSIFS + Tprop for waiting the CTS packet, NA will double its back off window and reinitiate to communicate with N3 by sending another RTS packet, i.e., the RTS2 packet. In the meantime, NB and NC will update their corresponding NAV timers based on the newly issued RTS2 packet as in Fig. 1b. Consequently, NA will result in a great amount of useless retries of sending RTS packets, which prohibit NB and NC from contending the channel and lead to the degradation of network throughput.

\section{Throughput Analysis For Art Protocol}

Throughput analysis will be performed to provide the mathematical modeling of proposed ART protocol. For this a two-dimensional Markov chain is used to describe the state transition of a node, where the state of each node is composed by the current retransmission stage and the current back off window size. Every data packet will be transmitted if the back off window size is counted down to zero value. Let the probability $p$ denote a source node that fails in transmitting its packet; while $(1-p)$ indicates the successful transmission probability. The two-dimensional Markov chain will return to its initial state if packet is successfully transmitted in each node. Otherwise, each node will increment its retransmission stage by one, and randomly determine its current back off size from the corresponding contention window size based on the BEB mechanism. Consider a saturated node that always has packets to transmit, the stationary transmission probability $\tau$ at a randomly selected time slot can be obtained from the two-dimensional Markov chain as

$$
\tau=\frac{2(1-2 \mathrm{P})}{(1-2 \mathrm{P})(\mathrm{W} 0+1)+\mathrm{PW} 0\left[1-(2 \mathrm{P})^{\mathrm{M}}\right.}
$$

where w0 denotes the minimal contention window size and $\mathrm{m}$ is maximum number of retransmissions. Note that the parameter $\tau$ can also be translated as the probability that a node will transmit a frame in a given time slot; while $(1-\tau)$ represents the probability for a node to remain silent. Therefore, the relationship between $\tau$ and $\mathrm{p}$ can be adopted to other random access-based MAC protocol with saturated nodes, i.e., it can be applied to the proposed ART scheme. To solve this nonlinear equation, an additional relationship between $\mathrm{p}$ and $\tau$ should be acquired

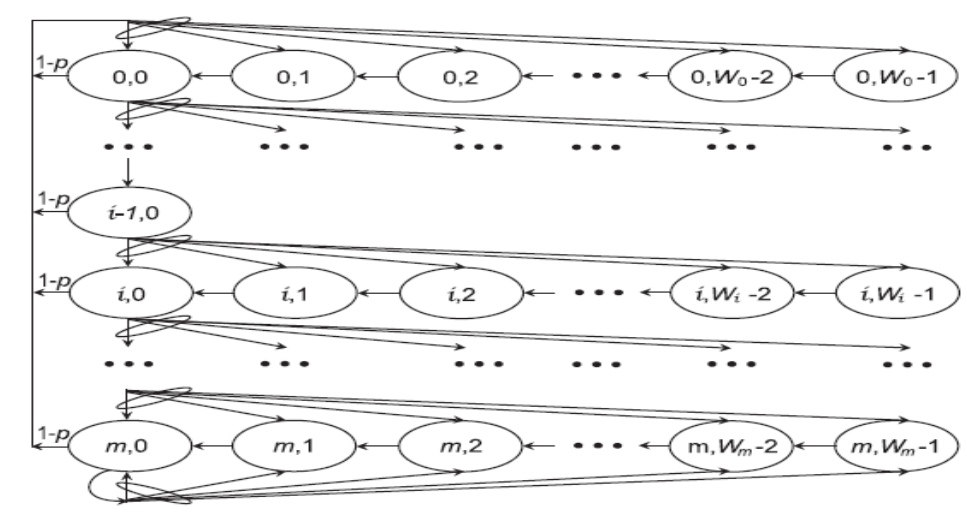

Fig 2 Two-dimensional Markov chain for contention-based state

such that both values can be solved by adopting numerical methods. In the following sections, how the stationary transmission probability $\tau$ affects the parameter $\mathrm{p}$ will be investigated in multihop ad hoc network with the existence of hidden terminals. 


\section{Introduction}

\section{Design Environment}

Network Simulator (Version 2), widely known as NS2, is simply an event-driven simulation tool that has proved useful in studying the dynamic nature of communication networks Simulation of wired as well as wireless network functions and protocols (e.g., routing algorithms, TCP, UDP) can be done using NS2. In general, NS2 provides users with a way of specifying such network protocols and simulating their corresponding behaviors.

Due to its flexibility and modular nature, NS2 has gained constant popularity in the networking research community since its birth in 1989. Ever since, several revolutions and revisions have marked the growing maturity of the tool, thanks to substantial contributions from the players in the field. Among these are the University of California and Cornell University who developed the REAL network simulator, the foundation which NS is based on. Since 1995 the Defense Advanced Research Projects Agency (DARPA) supported development of NS through the Virtual Inter Network Test bed (VINT) project. Currently the National Science Foundation (NSF) has joined in the development.

\section{Basic Architecture}

Figure 2 shows the basic architecture of NS2. NS2 provides users with executable command ns which ake on input argument, the name of a Tcl simulation scripting file. Users are feeding the name of a Tcl simulation script (which sets up a simulation) as an input argument of an NS2 executable command ns. In most cases, a simulation trace file is created, and is used to plot graph and/or to create animation.

NS2 consists of two key languages: $\mathrm{C}++$ and Object-oriented Tool Command Language (OTcl). While the $\mathrm{C}++$ defines the internal mechanism (i.e., a backend) of the simulation objects, the OTcl sets up simulation by assembling and configuring the objects as well as scheduling discrete events (i.e., a frontend). The $\mathrm{C}++$ and the OTcl are linked together using TclCL. Mapped to a $\mathrm{C}++$ object, variables in the OTcl domains are sometimes referred to as handles. Conceptually, a handle (e.g., n as a Node handle) is just a string (e.g.0 10) in the OTcl domain, and does not contain any functionality. Instead, the functionality (e.g., receiving a packet) is defined in the mapped $\mathrm{C}++$ object (e.g., of class Connector). In the OTcl domain, a handle acts as a frontend which interacts with users and other OTcl objects. It may defines its own procedures and variables to facilitate the interaction. Instant procedures (instprocs) and instance variables (instars), respectively. Before proceeding further, the readers are encouraged to learn $\mathrm{C}++$ and OTcl languages.

NS2 provides a large number of built-in $\mathrm{C}++$ objects. It is advisable to use these $\mathrm{C}++$ objects to set up a simulation using a Tcl simulation script. However, advance users may find these objects insufficient. They need to develop their own $\mathrm{C}++$ objects, and use a Otcl configuration interface to put together these objects.

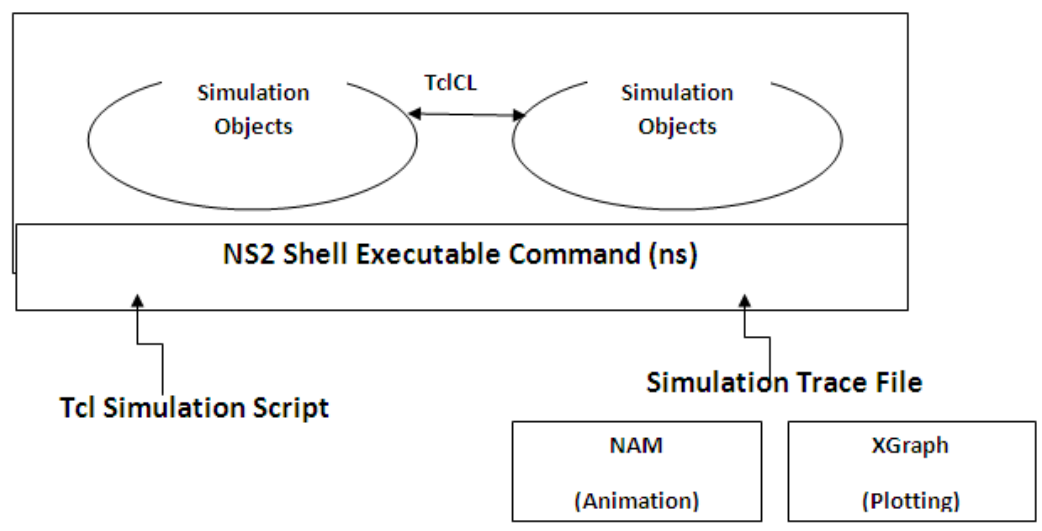

Fig 3 Basic Architecture of NS2.

After simulation, NS2 outputs either text-based or animation-based simulation results. To interpret these results graphically and interactively, tools such as NAM (Network AniMator) and Xgraph are used. To analyze a particular behavior of the network, users can extract a relevant subset of text-based data and transform it to a more conceivable presentation.

\section{Performance Evaluation}

\section{Results And Discussions}

The performance of proposed schemes will be evaluated and compared via custom event-triggeredbased $\mathrm{C} / \mathrm{C}++$ simulator. The simulator is composed of four major parts, including Node Class, Deployment Class, Scheduler Class, and Event Class. Node Class records current state of a node, e.g., back off stage, NAV 
value; while locations of all nodes are generated by Deployment Class. Event Class describes an event and the corresponding occurrence time, which is counted from current time. For example, if a node sends an RTS packet, the events will be triggered such that the neighbor nodes receive the RTS packet and the corresponding occurrence time is equal to Tprop + TRTS. Scheduler Class describes a scheduler which contains a timer and an event queue. The timer is employed to maintain current time; while all events are inserted to the event queue according to the addition of current time and their occurrence time. The event in the head of queue will be executed first and removed after execution has been completed. This event triggered- based simulator is considered feasible and is capable of simulating most of the network scenarios for ad hoc networks.

Saturation queue is considered in each network nodes for the purpose of evaluating the worst case of network scenarios. In general cases, the sensors will only occasionally report their measurements under normal environment. However, it is required for the sensors to frequently report their measurements for a period of time especially under severe network environments, e.g., earthquake. During this time period, the queues of sensors are considered approximately saturated.

The default settings for the simulation parameters are listed in Table 5.1. Note that some of the parameters are adopted from IEEE 802.11a standard. The network nodes are randomly distributed in a B $\times$ B square meters area, where the parameter B is denoted as the boundary limit. Note that the value of node density $\rho$ can be obtained as $\rho=\mathrm{N} /(\mathrm{B})^{2}$ with $\mathrm{N}$ as the total number of nodes in the network. Moreover, both the MAC header and the control packets, i.e., M-RTS, CTS, and ACK packets, are transmitted in basic rate; while the payload part of a data packet is delivered in data rate

\section{Performance Comparison}

In this section, the performance of proposed schemes will be compared with existing protocols including the conventional IEEE 802.11a DCF protocol, the MRT protocol. Fig. shows below the performance comparison between the Proposed protocols and the existing schemes. figure shows the performance comparison of throughput, packet delivery ratio and packet drop. As the total number of nodes in the network grows, it is intuitively to observe from the left plot that the throughput performance of all the schemes becomes worse because there can exist more packet collisions and additional interference from hidden nodes.

The proposed ART protocol can provide the highest throughput performance compared to the other schemes owing to its dynamic adjustment of selected receivers Mi. The proposed ART protocol still outperform the other methods with the highest throughput performance owing to its better channel utilization instead of constructing unnecessary connection attempts between the network nodes. Consequently, the simulation results show that the proposed ART protocols can consistently outperform the other algorithms and effectively alleviate the receiver blocking problem. The control overhead is defined as the number of RTS/MRTS Packets over the number of CTS packets,

\section{Simulation Results}

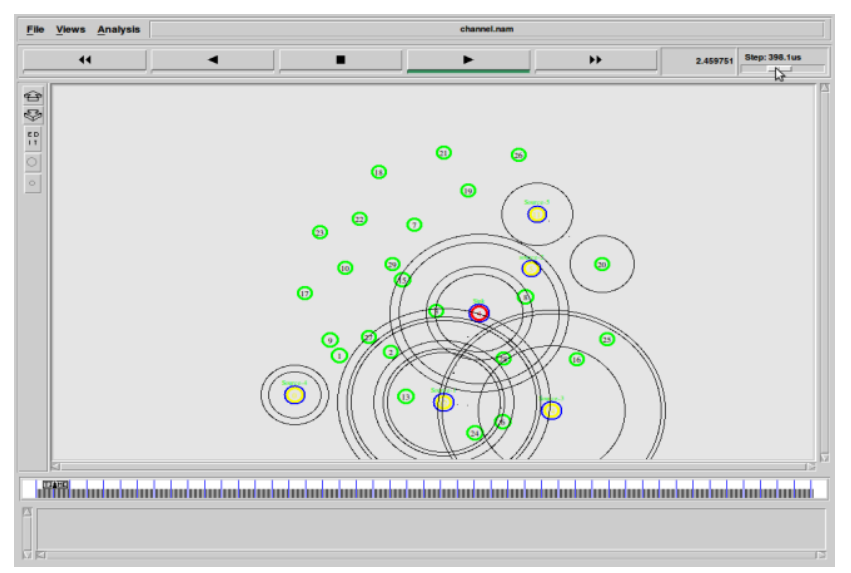

Fig 4 simulation shows data transmission in ad hoc network 


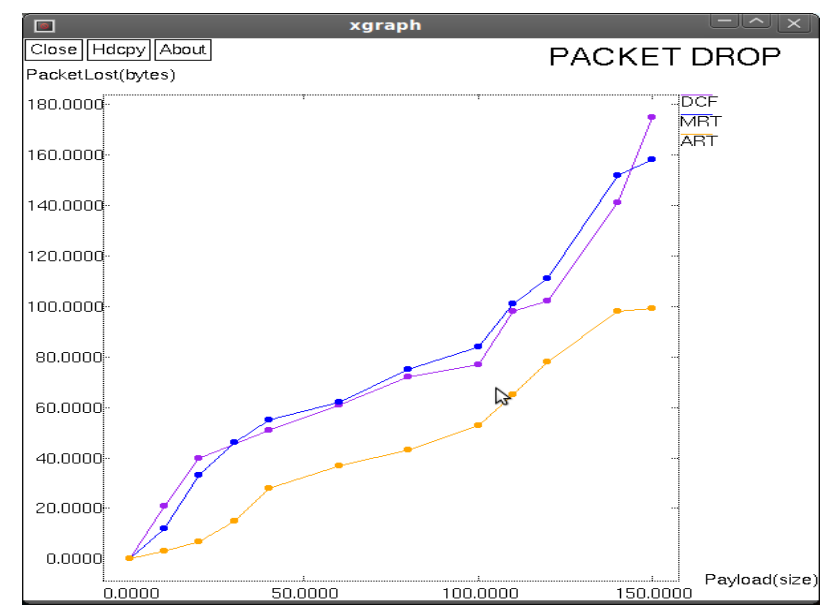

Fig 5 Simulation showing the packet drop

Comparison of packet drop in different scheme is shown here. Packet drop is less for ART protocol compared to other existing Mac protocols.

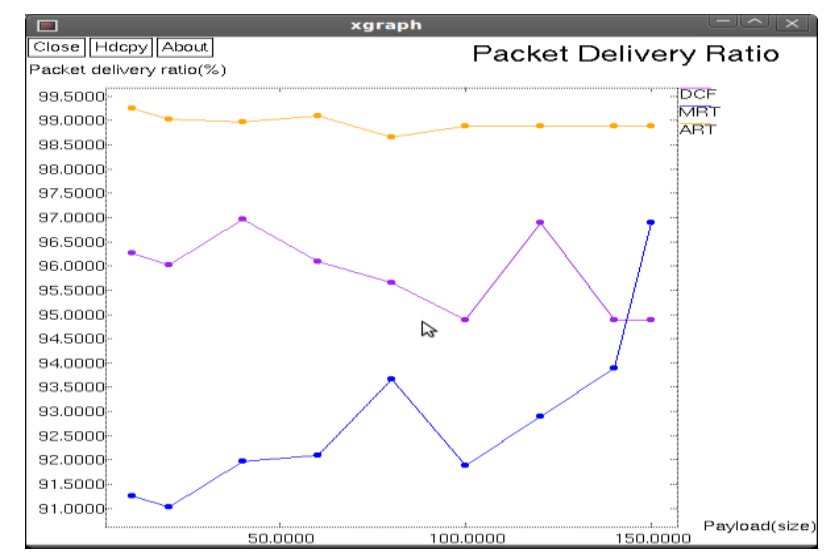

Fig 6 Simulation showing packet delivery ratio

Here comparison of packet delivery ratio of ART protocol with other existing Mac protocols is shown. Packet delivery ratio is more in ART protocol compared to other schemes.

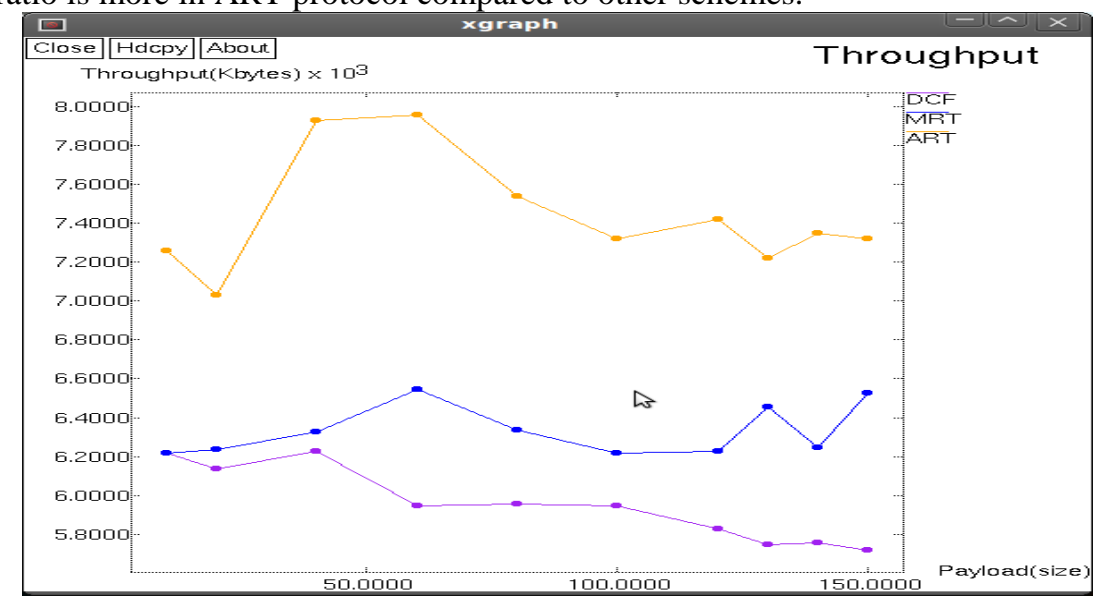

Fig 6 shows throughput performance

Here throughput of ART protocol is compared with other existing Mac schemes. Throughput is maximum for ART protocol compared to other schemes. This is the objective of this paper. 


\section{Inferences}

\section{Conclusion}

In this project, both the MRT and the FNT mechanisms are proposed to alleviate the receiver blocking problem in the multihop ad hoc networks. but it has some drawbacks. In M-RTS transmission, the data transmission delay of these selected receivers will be increased because each receiver will spend time waiting for the data packets that are not destined to itself until the end of the entire data transmission. The ART scheme is proposed to further improve the throughput performance with dynamic adjustment on the number of selected receivers. Thus the ART protocol alleviates the receiver blocking problem and improve the throughput performance more compared to other existing Mac protocols.

\section{Scope For The Future Work}

Going to implement the TDMA Scheduling scheme to improve the energy efficiency and reduce end to end delay. To achieve this objective, first build a nonlinear cross-layer optimization model involving the network, medium access control (MAC), and physical layers, which aims at reducing the overall energy consumption. Based on the network-wide flow distribution calculated from the optimization model and transmission power on every link, an algorithm called minimum delay scheduling algorithm is proposed for deriving the TDMA schedules

\section{References}

[1]. Alizadeh-Shabdiz.F and S.Subramaniam.S (2004), “Analytical Models for Single-Hop and Multi-Hop Ad Hoc Networks," Proc. IEEE First Int'l Conf. Broadband Networks (BroadNets), pp. 449-458

[2]. Alizadeh-Shabdiz.F and Subramaniam.S (2004), "MAC Layer Performance Analysis of Multi-Hop Ad Hoc Networks," Proc. IEEE GlobeCom, pp. 2781-2785

[3]. Chayabejara.A, S.M.S. Zabir, and N.Shiratori (2003), "An Enhancement of the IEEE 802.11 MAC for Multihop Ad Hoc Networks," Proc. IEEE 58thVehicular Technology Conf. (VTC-Fall), pp. 3020-3024

[4]. Chen.J, S.T.Sheu, and C.A.Yang (2003), "A New Multichannel Access Protocol for IEEE 802.11 Ad Hoc Wireless LANs," Proc. IEEE $14^{\text {th }}$ Personal, Indoor and Mobile Radio Comm. (PIMRC), pp. 2291- 2296

[5]. Ghaboosi.K, M. Latva-aho, Y.Xiao, and Q.Zhang (2009), "eMAC-A Medium Access Control Protocol for the Next Generation Ad Hoc Networks,” IEEE Trans. Vehicular Technology, vol. 58, no.8, pp. 4476- 4490

[6]. Hou.T.C, L.F.Tsao and H.C.Liu (2003), "Analyzing the Throughput of IEEE 802.11 DCF Scheme with Hidden Nodes," Proc. IEEE $58^{\text {th }}$ Technology Conf. (VTC-Fall), pp. 2870-2874 